\title{
Tracing the structural origins of atypical language representation: consequences of prenatal mirror-imaged brain asymmetries in a dizygotic twin couple
}

\author{
Anna-Lisa Schuler ${ }^{1} \cdot$ Lisa Bartha-Doering ${ }^{1} \cdot$ András Jakab $^{3} \cdot$ Ernst Schwartz $^{2} \cdot$ Rainer Seidl $^{1} \cdot$ Patric Kienast $^{2}$. \\ Sonja Lackner ${ }^{1} \cdot$ Georg Langs ${ }^{2}$. Daniela Prayer $^{2} \cdot$ Gregor Kasprian $^{2}$
}

Received: 8 October 2017 / Accepted: 13 July 2018 / Published online: 30 July 2018

(c) The Author(s) 2018

\begin{abstract}
We investigated the predictive value of prenatal superior temporal sulcus (STS) depth asymmetry in a special case of a female dizygotic twin that showed inverted prenatal asymmetry of this structure. For this purpose, we performed a follow-up investigation in this former fetus at the age of seven, where we assessed the functional language lateralization using taskbased and resting-state functional magnetic resonance imaging (fMRI). As control group we employed her twin brother, who showed a typical folding pattern prenatally, as well as a complementary set of four age-matched children that had fetal MRI of their brains and typical STS depth asymmetry. We could show that the twin with the atypical fetal asymmetry of the STS also showed significantly differing rightward language lateralization in the frontal and temporal lobes. Additionally, resting-state data suggest a stronger connectivity between inferior frontal gyri in this case. The twin showed normal cognitive development. This result gives a first glimpse into the STS' atypical asymmetry being a very early morphological marker for later language lateralization.
\end{abstract}

Keywords Atypical brain development $\cdot$ Fetal MRI · Functional MRI $\cdot$ Hemispheric specialization $\cdot$ Human language

\section{Introduction}

Electronic supplementary material The online version of this article (https://doi.org/10.1007/s00429-018-1717-y) contains supplementary material, which is available to authorized users.

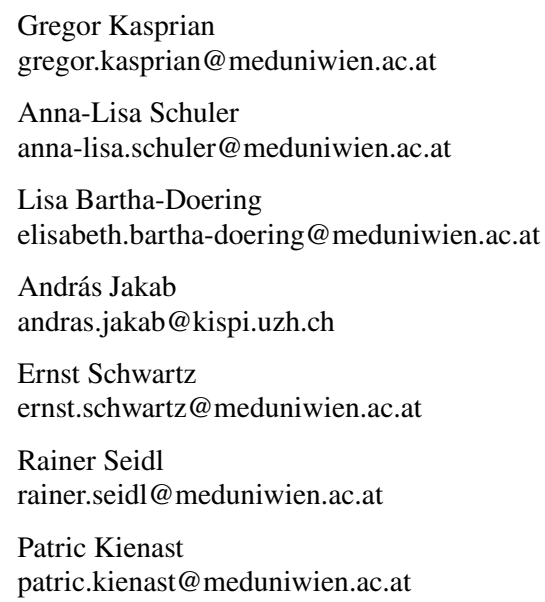

The associations between emerging asymmetry patterns of the human cerebral cortex observed on a structural and functional level have been the subject of an ongoing debate in clinical and cognitive neuroscience and have remained enigmatic to date.

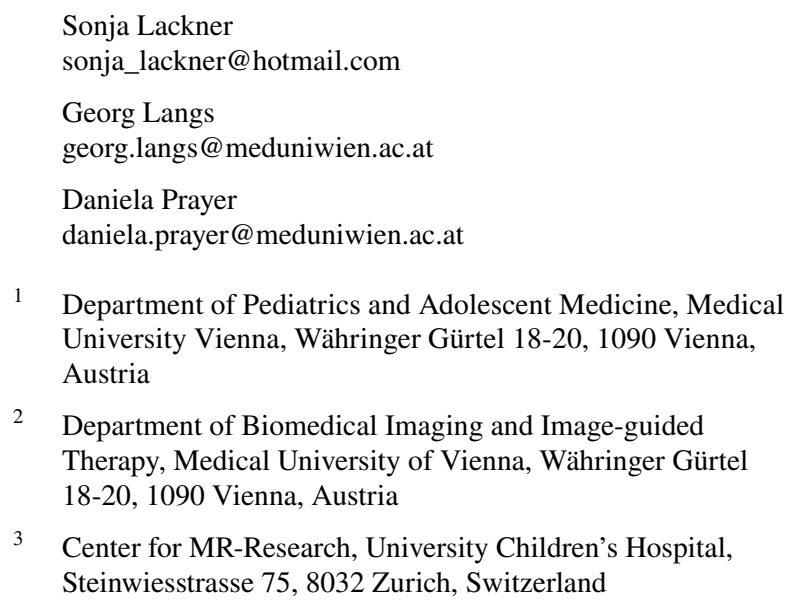

2 Department of Biomedical Imaging and Image-guided Therapy, Medical University of Vienna, Währinger Gürtel 18-20, 1090 Vienna, Austria

3 Center for MR-Research, University Children's Hospital, Steinwiesstrasse 75, 8032 Zurich, Switzerland 
The first associations between brain structure and function of language processing were made as early as in the nineteenth century. French neurologists Jean-Baptiste Bouillaud, Marcel Dax and Paul Broca blazed the trail for language and its laterality research based on their postmortem observations on aphasic patients with focal brain lesions to the frontal lobes.

The Austrian neuroanatomist Meynert (1868) was then the first to show that lesions to the temporal lobes are also involved in impaired language. An integration of these observations led his student Wernicke (1874) to draft the first model on the neural network of cognitive processing. On the contrary to Broca's articulatory aphasia, he describes a sensory aphasia that is caused by lesions to the temporal lobes.

Later on, in the twentieth century, the neuroscientist Norman Geschwind proposed the asymmetry of the temporal plane in the human brain (Geschwind and Levitsky 1968) and identified the inferior parietal lobule as an additional important player for language processing (Geschwind and Galaburda 1985).

These 200 years of language research led to the establishment of a modern dorsal and ventral stream model, with the dorsal stream being strongly involved in articulation and the ventral stream in encoding and retrieval of semantic contents (Poeppel 2014).

While this model is today considered as accurate for the mature brain, one important question remains to be uncovered in neurolinguistic research. It is the question how these functional-structural areas are being established at the first place.

The pursuit of potential early morphological markers for language processing just emerged with the recent establishment of more precise imaging methods in the human fetus and newborn.

The most promising markers for healthy fetal brain development are the time-sensitive gyrification patterns of the cerebral cortex since they serve as a marker for proper organization of cortical layers (Dobyns 1987). In this respect, Dubois et al. (2008) found the cortical folding patterns at preterm to be predictive for neural and neurocognitive development at term equivalent.

Typical age-sensitive sulcal development and asymmetry patterns were first established by postmortem studies (Dorovini-Zis and Dolman 1977; Chi et al. 1977; Wada et al. 1975). In in vivo in utero studies, it became evident that the development of the human fetal superior temporal sulcus (STS) occurs at different time points, with the right STS forming around 2 weeks earlier than the left (Kasprian et al. 2011; Habas et al. 2012; Rajagopalan et al. 2011). This initial asymmetry was recently confirmed to persist throughout life, as reflected by the so-called superior temporal asymmetrical pit (Leroy et al. 2015). This feature was recently brought into a broader context by identifying a whole human-specific brain torque around the Sylvian fissure, which is probably at the core of developmental asymmetries of the superior temporal sulcus ( $\mathrm{Li}$ et al. 2018).

Recently, Kersbergen et al. (2016) were able to identify the configuration of the early superior temporal sulci as predictive marker for receptive language. They investigated the cortical folding patterns in extremely preterm born neonates between the 24th and 28th week of gestation and at a followup investigation at the ages between 24 and 30 months. At their follow-up, receptive language was significantly correlated, with sulcal depth of superior temporal sulci.

On a functional level, language turned out to be clearly lateralized in the mature human population (Ocklenburg et al. 2014). While most humans show a leftward bias of language lateralization, some individuals show atypical language lateralization to the right hemisphere or no specialization at all (Knecht et al. 2000).

Considering these parallels in structural and functional asymmetries, many studies aimed to unravel the mutual precursors of the two patterns with, to date, inconclusive results (Tzourio et al. 1998; Josse et al. 2003, 2009; Dorsaint-Pierre et al. 2006; Greve et al. 2013; Keller et al. 2011). Despite the advancements in language research provided by those studies, there were no predictive statements to be derived, because they were based purely on correlational analyses.

Since models are considered to be more robust if they can make predictive statements, we aimed to introduce a prenatal morphological marker into the ongoing debate on laterality that would enable an investigation of the specific dynamics between emerging structural brain asymmetries and postnatal functional specialization. We chose the brain asymmetry within the temporal lobes-best represented by the hemispheric difference in STS depth at around 24-28 gestational weeks in the human fetus (Kasprian et al. 2011), as a potential marker for postnatal language lateralization. This is the earliest fetal cortical marker for asymmetry of the human brain to be observed.

In adults' fMRI studies, the STS was found to play a role in language processing (Deen et al. 2015; Fedorenko et al. 2012; Fedorenko and Thompson-Schill 2014; Vigneau et al. 2006; Binder et al. 1997). This area was identified as a part of the phonological network and was specifically associated with the analysis of changing sequences of sensory input (Poeppel 2014; Redcay 2008). Furthermore, Lohmann et al. (2009) found consistent task-based connectivity over different language paradigms between a left inferior frontal gyrus seed region (BA44) with the STS. Beyond these correlational observations, the STS was recently shown to be at the cerebral basis of the McGurk effect, as revealed by specific manipulation through transcranial magnetic stimulation (Beauchamp et al. 2010). 
Our potential marker, the STS depth asymmetry, is rightward oriented in about $97 \%$ of healthy fetuses. Therefore, we suppose that the small percentage of atypical fetuses has a special value on prognostics of typical and atypical language and general neurodevelopmental organization. In this respect, we managed to evaluate the language asymmetry in a very rare case of pronounced leftward depth asymmetry of the STS occurring in a, to the date of the follow-up study, 7-year-old girl. As controls we assessed language lateralization in (1) her twin brother, who had typical STS depth asymmetry, (2) a complementary sample of four agematched children that also showed typical STS depth asymmetry in fetal MRI of their brains.

The first aim of the present study was to unfold neural differences in language lateralization between a case of atypical, asymmetrical prenatal brain development to a typical case matched for environmental conditions, namely the twin brother.

Second, we wanted to detect differences between the subject exposing atypical STS asymmetry and a group of typical fetal, age-matched controls in terms of language lateralization and localization.

Finally, we investigated how differences in language laterality translate to a general network level by comparing language-related, resting-state networks in both twins at 7 years of age (McAvoy et al. 2016).

By investigating a deviation from normal cortical folding patterns, this paper describes the first step toward understanding the co-evolution of functional and structural brain development, particularly as it covers one investigation at a mid-fetal stage and another at the age of seven.

The results of this study will give a first glimpse into the prenatal origins of language development, complementing existing work on infants born preterm (Mahmoudzadeh et al. 2013), and inform future studies on this topic. More finegrained prenatal diagnostics will arise based on this research enabling novel intervention strategies for at-risk fetuses.

\section{Materials and methods}

\section{Participants}

The female twin that was selected for the study due to her atypical STS depth asymmetry prenatally was at the time of follow-up a 7.8-year-old right-handed [EHI = 100; (Oldfield 1971)] native German speaker. At the time of fetal MRI, viz. at $28+2$ weeks of gestation, she demonstrated atypical asymmetry of the STS, i.e., a deeper left and a shallower right. Both twins showed no signs of any brain pathology at the time of fetal MRI. She and her twin brother were delivered preterm at $32+0$ weeks of gestation via Cesarean section because of maternal pre-eclampsia, and spent 3 days at the neonatal intensive care unit. The following 2 months they spent in a thermal bed. In view of the circumstances, postnatal development was considered normal. At the time of cognitive testing, she and her brother attended the Austrian elementary school (Volksschule), first grade. Both twins showed an age-appropriate profile in their cognitive skills, as assessed by a comprehensive in-house neuropsychological test battery (Table S1). They have no other siblings and their parents are both right-handed, native German speakers.

The heterozygotic, twin brother with typical fetal STS depth asymmetry was included in the study as a control case, together with a group of four age-matched controls (all 7 years old, two female). They were all native German speakers with a fetal asymmetry index of $<0$ (marking a typical fetal lateralization pattern, see Table S2), were at a sensitive developmental stage for fetal STS asymmetry measurement, and had no neurological diagnosis, based on the fetal MRI, or to the date of follow-up assessment (see Table S2). In addition, they did not demonstrate familial left-handedness.

The study protocol was approved by the local ethics committee of the Medical University of Vienna, Austria. Informed consent was obtained from all individual participants included in the study. All participants provided informed written consent by their legal guardian and received reimbursement in the form of a voucher for a bookshop. The study was performed in accordance with the Declaration of Helsinki (Rickham 1964) and its later amendments.

\section{Fetal MRI data acquisition}

Fetal magnetic resonance imaging (MRI) was performed on a Philips 1.5 T superconducting unit (Philips Gyroscan) at the Department of Radiology, Division of Neuroradiology, Medical University of Vienna. A SENSE cardiac coil, with five elements (three posterior and two anterior) was wrapped around the mother's abdomen and re-adjusted during the imaging procedure, depending on the position of the fetal head. Routine fetal imaging required between 30 and $45 \mathrm{~min}$ (with approximately $20 \mathrm{~min}$ of dedicated fetal neuroimaging). Neither the mother nor the fetus was sedated. Fetal neuroimaging consisted of single-shot, fast spinecho T2-weighted sequences (TE/TR: 100/1400 ms; slice thickness: $3 \mathrm{~mm}$; field of view: $200 \mathrm{~mm}$; matrix $256 \times 256$; in-plane resolution: 0.78 pixels per $\mathrm{mm}$; flip angle: $90^{\circ}$; acquisition time $15 \mathrm{~s}$ ), acquired in three orthogonal planes. Sequence preparation and planning of orthogonal orientations were guided by an anatomist (Kasprian et al. 2011) or by an experienced fetal radiologist (D. P., G. K.), both with substantial expertise in the field of fetal neuroimaging. Axial images were acquired perpendicular to the axis of the fetal 
brain stem and served as a reference for the exact anatomical preparation of the coronal sequences, which were planned orthogonally, passing through corresponding positions of both inner ears and following the angle of the fetal brain stem, as depicted by sagittal T2-weighted sequences.

\section{Follow-up fMRI data acquisition}

At 7 years of age, functional magnetic resonance imaging (fMRI) was performed on a $3 \mathrm{~T}$ Tim Trio (Siemens Medical Solutions, Erlangen, Germany) whole-body MR tomograph, using the manufacturer's default, 12-channel RF head coil. The functional $\mathrm{T} 2 *$ scans were performed using a phasecorrected, blipped gradient echo, single-shot echo planar imaging (EPI) sequence (TE/TR: $42 / 2000 \mathrm{~ms}$, slice thickness: $4 \mathrm{~mm}$; field of view: 210 ; voxel size: $2.1 \times 2.1 \times 4 \mathrm{~mm}^{3}$; 25\% gap; 20 axial slices parallel to the AC-PC plane; flip angle: $90^{\circ}$ ). Five minutes of resting-state fMRI EPI were acquired using the same scan parameters as for the abovementioned language paradigm. We acquired additional T1-weighted anatomical images that were not included in further analyses.

\section{Paradigm}

During fMRI assessment, an auditory word-definition task was applied, to measure the neural correlates of higher order language processing. In healthy, right-handed children, this paradigm has shown robust language lateralization to the left hemisphere, including the inferior frontal gyrus, the middle temporal gyrus, the supplementary motor cortex, the middle frontal gyrus, and the fusiform gyrus (Balsamo et al. 2006; Gaillard et al. 2007; You et al. 2011). This paradigm requires comprehension of a phrase, semantic recall, and a semantic decision, includes a button-press response, and is suitable for children 5 years of age and above. During the active condition, participants listened to a definition of an object, followed by a noun. The children were instructed to press a button each time they judged that the description matched the noun. For instance, "a long yellow fruit is a banana" (true response) or "something you sit on is a spaghetti" (not true). Definitions occurred every $3 \mathrm{~s}$. Matching pairs were pseudo-randomly distributed (70\% true responses, $30 \%$ foils). During the control condition, task definitions were presented in reverse speech. Participants were instructed to press a button each time they heard a tone after the auditory string ( $70 \%$ true responses, $30 \%$ foils). The baseline was designed to control for first- and second-order auditory processing, attention, and motor response (You et al. 2011). Nouns were selected according to linguistic criteria that included word frequency, word length, and word complexity and were well balanced within five semantic categories. Three different difficulty levels were available for the present study, and the age-appropriate difficulty level of tasks, i.e., simple, was chosen.

\section{Language fMRI data preprocessing}

Data preprocessing included realignment, segmentation, normalization to MNI space, and $6 \mathrm{~mm}$ spatial smoothing performed with a FWHM filter, as implemented in SPM12: linear regression at each voxel using generalized least squares with a global approximate AR(1) autocorrelation model, drift fit with discrete cosine transform basis (128 s cutoff) resulted in $t$ maps on a single-subject level. Subsequently, a contrast was calculated between conditions forward speech $>$ reverse speech $(p<0.05$, family-wise error corrected).

For group comparison, a two-sample, second-level $t$ test was performed with the female twin with atypical prenatal asymmetry in the first group and the typical controls in the other group. The $t$ map was cluster-level corrected at $p<0.05$, family-wise error corrected, with an initial threshold of $p<0.001$, uncorrected.

To check if there is any residual effect of phonemic or prosodic information in the reverse speech condition that could have influenced laterality estimations, we calculated additional one-sample $t$ maps for forward speech $>$ reverse speech and reverse speech $>$ forward speech for the control sample. These $t$ maps were also cluster-level corrected at $p<0.05$, family-wise error corrected, with an initial threshold of $p<0.001$, uncorrected.

\section{Laterality indices}

The fetal laterality index (LI) was calculated using the ratio of the left-right to the left + right sulcal depth. In the case of fetal STS depth, an experienced neuroradiologist (G. K.) quantified this measure in $\mathrm{mm}$. For this purpose, a straight line between the two bulges of the emerging superior and middle temporal gyri was drawn. In a second step, an orthogonal line was drawn from between this line to the deepest pit of the emerging STS. This method was validated with an unsupervised computational model (Kasprian et al. 2011) and recently shown to be appropriate by interrater comparison (Schuler et al. 2017).

Concerning functional data, the preprocessed $t$ maps for the first-level contrast forward speech $>$ reverse speech were fed into the default, bootstrapping approach, as implemented in the LI toolbox for SPM12 (Wilke and Lidzba 2007). Lateralization of activations was estimated on the single-subject level. A frontal, temporal, and parietal region of interest (as provided by the LI toolbox) was used as an inclusive mask, as well as a whole-brain gray matter brain mask ROI. To avoid the threshold dependency of simple lateralization indices, a bootstrapping approach was employed (Lidzba et al. 2011). 
With this approach, a multitude of bootstrapping resamples from the original dataset could be analyzed at different thresholds, yielding a single, weighted mean laterality index (LI), which was based on the whole of the underlying dataset (Wilke and Schmithorst 2006). Functional LIs were categorized as left-lateralized if $\mathrm{LI} \geq 0.2$, bilateral if $\mathrm{LI}$ was within -0.2 and +0.2 , or right if $\mathrm{LI} \leq-0.2$. For case-to-small-group comparison, Crawford's adapted $t$ test was used (Crawford and Howell 1998). $t$ tests were calculated to investigate significant differences in frontal, temporal, and parietal laterality between the atypical case and the small reference group of typical former fetuses.

\section{Resting-state fMRI data processing}

The acquired resting-state data were processed using an opensource optimized pipeline (https://github.com/janeisklar/ RSTools). During preprocessing, data were de-spiked using AFNI (http://afni.nimh.nih.gov/afni). To compensate for slight time lags between acquisition of slices for a 3D EPI, temporal sinc interpolation of the MR signal was used, by shifting the signal of the misaligned slices back in time to the reference slice using FSL 5 (FMRIB Software Library, Analysis Group, FMRIB, Oxford, http://fsl.fmrib.ox.ac.uk). Data were realigned using FSL 5, normalized to standard MNI space using ANTs in combination with a custom, scanner-specific EPI-template that resulted in a $1.5 \mathrm{~mm}^{3}$ isotropic resolution, and, finally, smoothed with a 6-mm FWHM Gaussian kernel using FSL 5. The post-processing was carried out using programmer-optimized applications, which were mostly based on the GNU Scientific Library (http://www.gnu.org/software/gsl) and comprised the following steps: (1) regressing out the cerebrospinal fluid and white matter signal to reduce physiological artifacts using the first five components of a temporal PCA and the mean for both regions, as well as the global mean; (2) FFT-based band-pass filtering in the frequency range of 0.009-0.08 Hz; (3) motion-scrubbing according to (Power et al. 2012); and (4) seed voxel correlation using the mean time course of language-related fMRI seeds of the auditory word-definition task, based on the results from a group $t$ map of 28 healthy children $(16 \mathrm{~m}$, mean age: $9.82 \pm 2.37$ years, $\mathrm{min} /$ $\max =7 / 16$ years). For seed voxel correlation, a left $(-5030$ $10)$ and right (36 $30-10)$ IFG, as well as a left posterior STS $(-52-340)$ peak region and its contralateral homolog (52 -340 ) were selected, with $6 \mathrm{~mm}$ radii around the peak voxels. Single-subject correlation maps of Fisher $z$-transformed correlation strengths were calculated for both twins and all four networks.

\section{Results}

\section{Twin laterality differences}

The fetal MRI of the female twin revealed an atypical structural left-right asymmetry with a deeper left STS $(3 \mathrm{~mm})$ than the right STS $(1 \mathrm{~mm})$ at GW $28+2$. In contrast to this, her twin brother showed the opposite typical pattern with a deeper right STS $(4 \mathrm{~mm})$ and a shallower left STS $(2 \mathrm{~mm})$ in utero (Fig. 1a). At 7 years of age, the fMRI follow-up of the female twin exhibited overall atypical symmetric language processing, with a tendency toward an increased rightward activity (i.e., $<0$ and $>-0.20$ ), associated with language functions, during the auditory word-definition task [overall gray matter LI -0.17, SD 0.18; (Sepeta et al. 2016)]. Single-subject analysis of the female twin revealed increased activity in the bilateral, posterior, superior temporal sulcus, the right superior operculum of the IFG, the right posterior superior part of the pars triangularis of the IFG and the inferior posterior part of the rostral, middle frontal gyrus (Fig. 1c). The male twin showed an overall gray matter LI of 0.63 (SD 0.25 ), with significantly increased activity in the left posterior superior temporal sulcus, the bilateral, inferior frontal and left dorso-lateral prefrontal cortex, the bilateral anterior insulae, and the head of the left caudate nucleus, compared to his twin sister (Fig. 1c). A detailed LI comparison is shown in Table 1 and Fig. $1 b$.

\section{Group language laterality differences for the atypical twin}

The frontal $\left(t_{4}=-8.04, p=0.00065\right.$, one tailed; Crawford's $t$ test $)$ and temporal $\left(t_{4}=-8.08, p=0.000637\right.$, one tailed; Crawford's $t$ test) functional language LI of the atypical twin differed significantly from the age-matched control group with a typical rightward fetal asymmetry. The atypical twin did not differ significantly $\left(t_{4}=-1.75\right.$, $p=0.078$, one tailed; Crawford's $t$ test) from the control group in terms of parietal language lateralization.

\section{Group localization differences for the atypical twin}

To further explore the quality of language-related differences, a two-sample $t$ test between the atypically lateralized twin and the reference population was performed. The results revealed increased activity in the left conjunction between the parahippocampal, fusiform, and lingual gyri $(p<0.001$, MNI-peak: $-36-58-12, k=44)$ in the atypically lateralized twin compared to the typically lateralized fetal reference group (Fig. 2). 
a

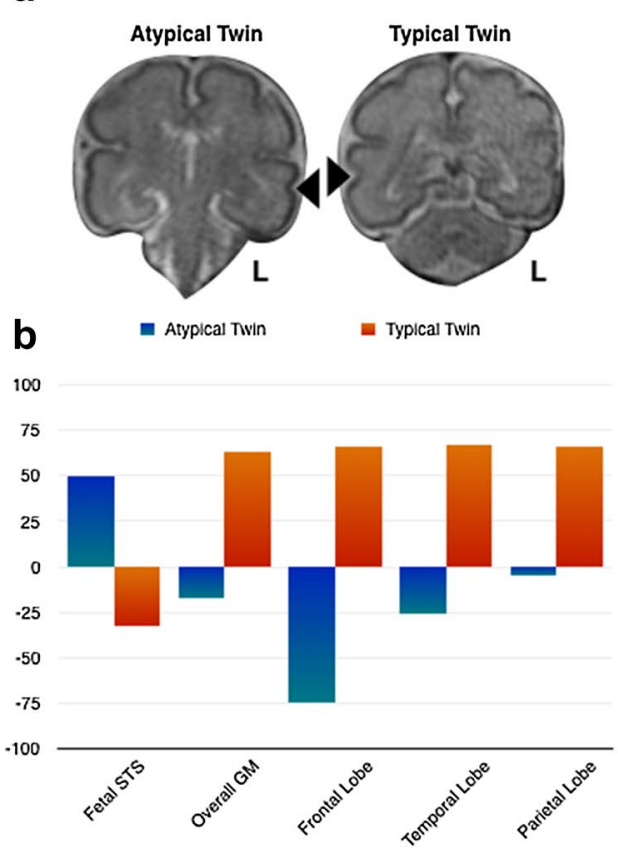

C
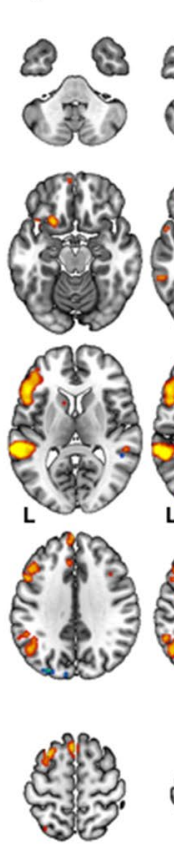
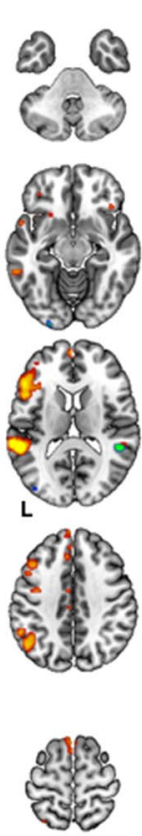
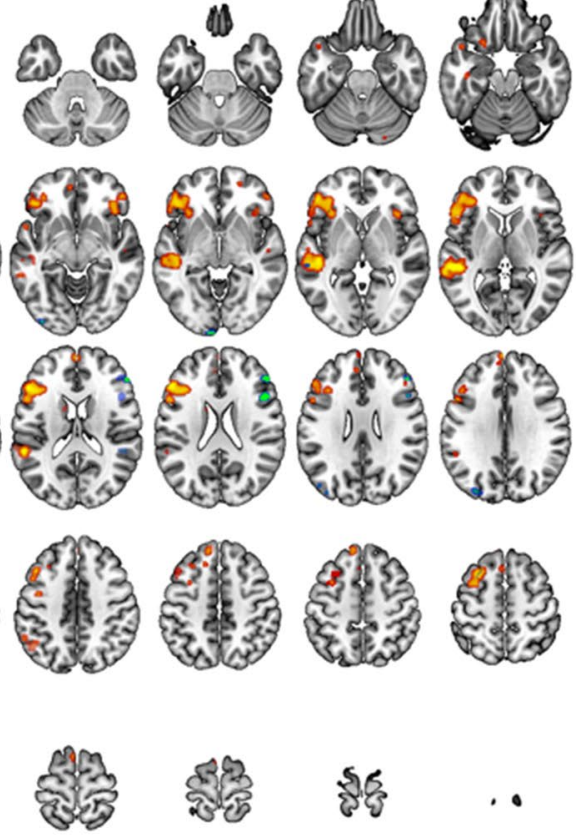

10

10

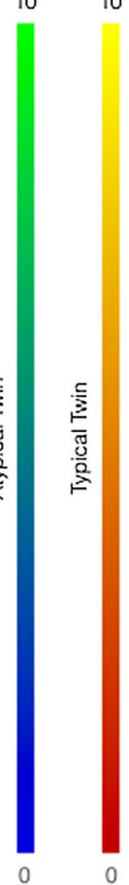

Fig. 1 Comparison of fetal asymmetries and language lateralization in 7-year-old twins. a The fetal MRI of the female twin revealed an atypical structural left-right asymmetry with a deeper left STS $(3 \mathrm{~mm})$ than the right STS $(1 \mathrm{~mm})$ at $\mathrm{GW} 28+2$. In contrast to this, her twin brother showed the opposite typical pattern with a deeper right STS $(4 \mathrm{~mm})$ and a shallower left STS $(2 \mathrm{~mm})$ in utero (see black arrow heads). The fetal images are depicted in radiological convention. b The fetal laterality index for the atypical twin is 50 and, therefore, considered leftward whereas the typical male's laterality index is -33 and, therefore, considered rightward. In terms of language lateralization the typical twin shows consistent leftward laterality (over-

Table 1 Comparison of twin laterality

\begin{tabular}{lll}
\hline Laterality & Female twin & Male twin \\
\hline Fetal STS & 0.5 & -0.33 \\
Overall GM & $-0.17(\mathrm{SD}=0.18)$ & $0.63(\mathrm{SD}=0.25)$ \\
Frontal & $-0.75(\mathrm{SD}=0.16)$ & $0.66(\mathrm{SD}=0.2)$ \\
Parietal & $-0.05(\mathrm{SD}=0.2)$ & $0.66(\mathrm{SD}=0.23)$ \\
Temporal & $-0.26(\mathrm{SD}=0.22)$ & $0.67(\mathrm{SD}=0.23)$ \\
\hline
\end{tabular}

\section{Twin connectivity differences}

To gain complementary insights into the functional architecture of the twins' brain states, seed voxel correlation was calculated for four language-related seeds: the left and right IFG and the left and right posterior STS. While the male twin had stronger intra-hemispheric connectivity of the left IFG seed voxels to the left posterior STS, the atypically lateralized twin sister showed increased connectivity to the left posterior STS and additionally increased all 63 , frontal 66, temporal 67, parietal 66) whereas the atypical twin shows rightward laterality in the frontal $(-75)$ and temporal lobe (26) and bilaterality in overall gray matter $(-17)$ and in parietal lobe (-5). c The atypical fetal twin (cold colors) also showed an atypical lateralization pattern in response to the language paradigm. She showed increased activity in the right IFG and the bilateral posterior STS. Her fetal typical brother (warm colors), however, showed typical lateralization to the left IFG and posterior STS, with bilateral IFG activation changes. $t$ maps were FWE corrected at a threshold level of $p<0.05$ and are displayed in neurological convention. Laterality indices were multiplied by 100 for visualization purposes

connectivity between the left IFG and the ipsilateral anterior cingulate cortex (ACC). Concerning the right IFG, the female twin demonstrated a widespread frontal network that encompassed enlarged, local connectivity around the target area, as well as the contralateral homologue and the bilateral orbito-frontal cortex. The typically lateralized twin brother showed only a low connectivity to the contralateral homologous area and connectivity spread to the right anterior insula, as well as to the right ACC. The atypically lateralized twin further showed increased connectivity between the left posterior STS and the bilateral anterior midline and its contralateral homologue. Both twins exhibited increased connectivity from the left posterior STS to the left IFG, but the typically lateralized twin demonstrated additional increased connectivity to the left inferior parietal lobule (IPL) and the left posterior hippocampus. The right STS network consisted of the contralateral left STS and IFG in the male twin; however, the female twin showed a much wider network consisting of 
Fig. 2 Significant differences in language localization between the atypical twin and the typical control group. Two-sample $t$ test revealed a significant cluster at the junction of the left parahippocampal, fusiform, and lingual gyri. The $t$ map was cluster-level corrected for $p_{\mathrm{FWE}}<0.05$ at an initial threshold level of $p<0.001$, uncorrected
Language Localization: atypical twin>group
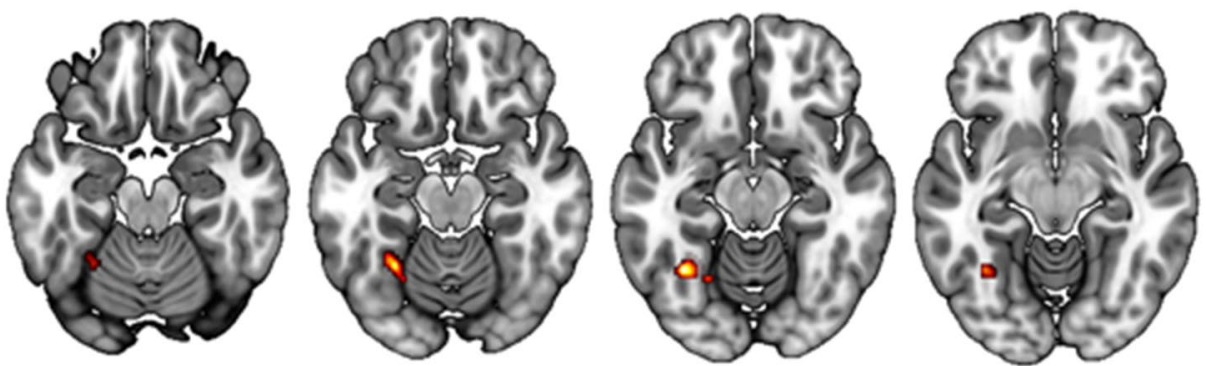
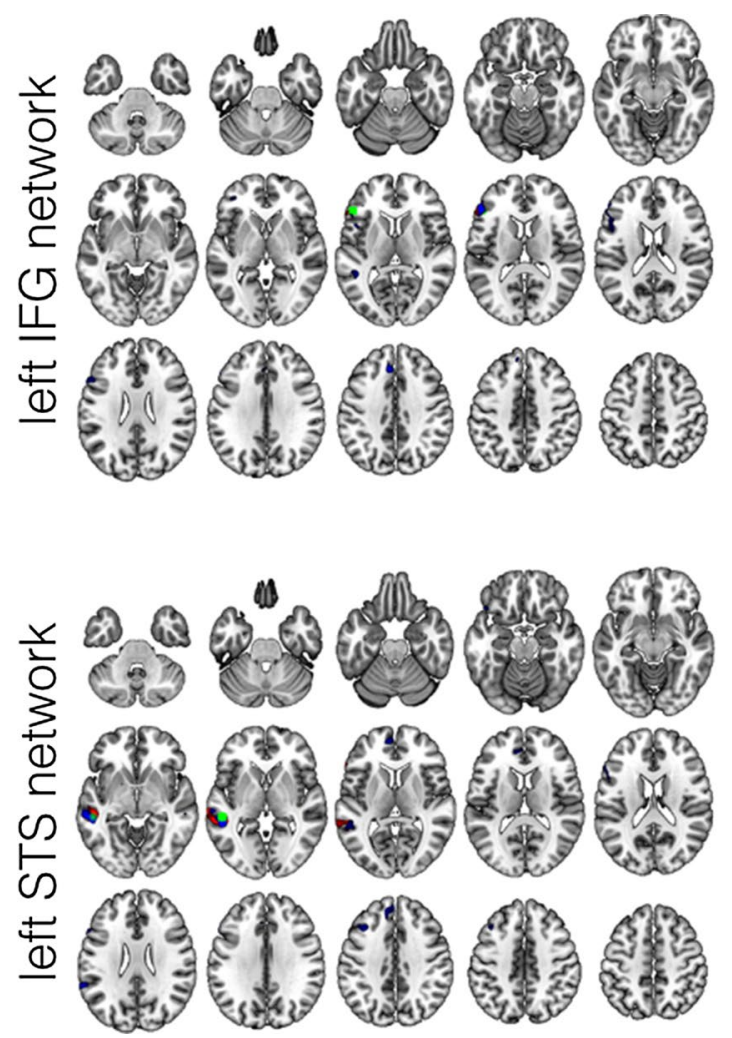

Fig. 3 Comparison between language-related networks between twins. Seed voxel correlation for the network that originated from the left IFG seed (top left) was performed using a 6-mm sphere around left IFG peak activity (MNI -50 30 10; green) of the functional localizer task. The male twin showed stronger intra-hemispheric connectivity of the left IFG seed to the left STS (red). The atypical twin sister also had connectivity to the ipsilateral STS area, as well as an additionally increased connectivity between the left IFG and the ipsilateral ACC (blue). Seed voxel correlation for the right IFG network (top right) was performed using a 6-mm sphere around the right IFG peak activity (MNI $3630-10$; green) of the functional localizer task. The male twin showed little contralateral connectivity, and right insula and right ACC connectivity (red). The atypical twin sister, however, showed strong contralateral IFG and bilateral OFC con-
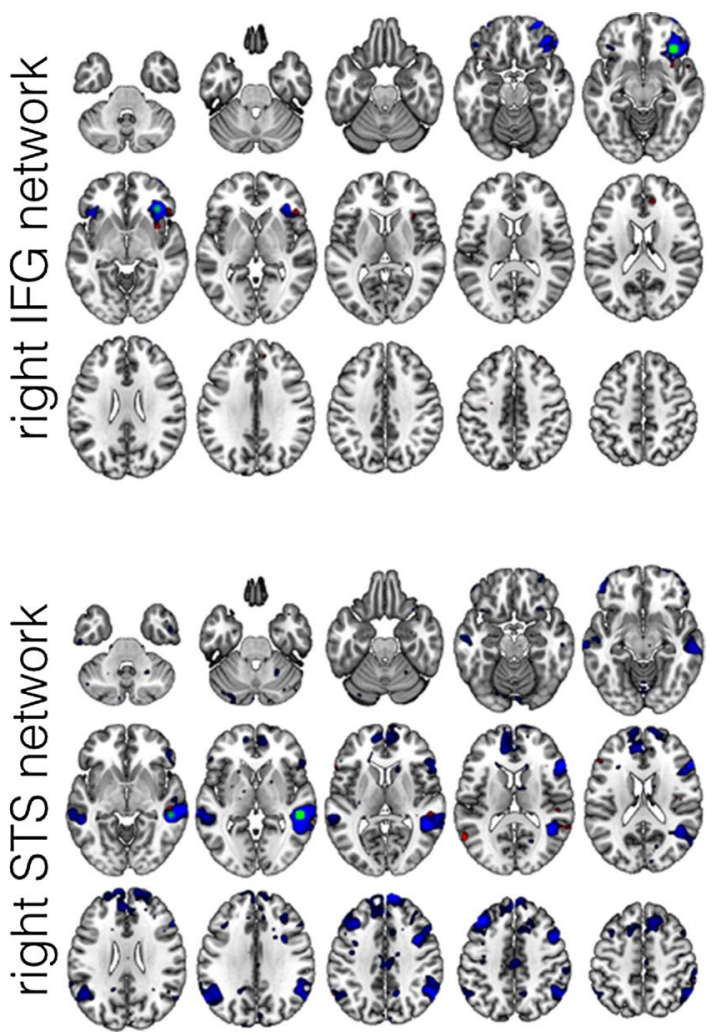

nectivity (blue). Seed voxel correlation for left posterior STS (bottom left) seeding network was performed using a 6-mm sphere around the left posterior STS peak activity (MNI $-52-340$; green) of the functional localizer task. The typical male twin showed connectivity to left IFG, IPL, and posterior hippocampus (red). The atypical twin sister showed connectivity to the left IFG, as well as to the posterior STS; however, she also showed stronger contralateral temporo-parietal junction (TPJ) connectivity, as well as left putamen and bilateral midline connectivity (blue). A seed voxel correlation (bottom right) for the right STS seed (MNI 52 - 34 0; green) revealed a correlation to the contralateral left STS and IFG in the male twin (red); however, the female twin (blue) showed a much wider network consisting of contralateral STS, bilateral IFG, OFC and anterior midline 
contralateral STS, bilateral IFG, OFC and anterior midline (Fig. 3).

\section{Additional group-map results}

The forward speech $>$ reverse speech group contrast revealed two significant clusters in the dorso-medial prefrontal cortex [MNI-peaks: -4 $3046(p<0.001)$; $-64230(p=0.049)$ ], left middle superior temporal sulcus ( $p<0.001$, MNI-peak: $-62-303$ ), hippocampus ( $p=0.049$, MNI-peak: $-34-38$ $-10)$ and ventral tegmental area $(p<0.001$, MNI-peak: 4 $-18-14)$. The reverse speech $>$ forward speech contrast, on the other hand, revealed increased activation in the precuneus ( $p<0.001$, MNI-peak: $12-7254)$ and supramarginal gyrus ( $p=0.027$, MNI-peak: $48-3636) . t$ maps are depicted in Figure S1.

\section{Discussion}

In this study, we describe atypical cortical folding patterns in the brain of a female twin fetus, coupled with strongly atypical language lateralization, localization, and connectivity in childhood, compared to a group of typical contemporaries including her twin brother. Although both twins exhibited asymmetries of the superior temporal sulcus (STS) prenatally, the female twin showed an atypical STS asymmetry, i.e., a deeper left STS and a shallower right STS as opposed to her brother. The configuration of rightward asymmetry of the STS has already been described in $97 \%$ of the cases in a larger sample of fetuses, and is, therefore, considered typical (Kasprian et al. 2011).

Since the difference between the children's asymmetry patterns around the temporal/perisylvian areas remains striking between mid-fetal age and the age of seven, we strongly suggest that genetic more than environmental differences underlie the differences in fetal cortical folding and consequent childhood language processing in the twin siblings. Converging recent evidence on asymmetric gene expression in the fetal temporal lobes (Pletikos et al. 2014; Miller et al. 2014; Sun et al. 2005) and hard-wired loci of languagerelated functional areas (Dehaene and Dehaene-Lambertz 2016) either suggests that the atypical language lateralization in our twin case is a direct consequence of atypical fetal STS configuration or that both-atypical fetal folding and atypical language localization-are driven by a common underlying genetic factor. This specific morphological marker seems to explain more reliably phenomena accessible on a behavioral level than life events or circumstances, as growing up in different environments, like rural or urban, or in a family with high or low socioeconomic status (Hackman and Farah 2009). We make this claim, because we controlled for environmental factors, in part, by comparing the atypical case to her typical twin brother, who shared not only the same uterine environment with his sister, but also the same life environment, including the same socioeconomic status of parents and attendance at the same kindergarten and elementary school.

An atypical configuration of the perisylvian area in dizygotic twins of different sexes has been described by Plante et al. (1989). Their report included a pair of dizygotic twins at 4 years of age, with the male twin demonstrating impaired language, coupled with symmetry of the perisylvian area, and the female twin not impaired linguistically, but demonstrating inversed asymmetry in the perisylvian area. We could also show different fetal structural, and, later, functional asymmetries in our bi-gendered twin couple; however, they showed a balanced cognitive profile without any linguistic impairments at 7 years of age.

Compared to the control group of typically lateralized children in their fetal state, the atypically lateralized female twin had significantly stronger rightward language lateralization in the temporal and frontal cortices. This difference in functionality might be a consequence of a difference in cell migration and proliferation during the fetal stage caused by individual differences in gene expression in the emerging neo-cortex. As Pletikos et al. (2014) suggest, there is a peak in specific gene co-expression in early mid-fetal development (16-19 post-conception weeks) in the superior temporal and primary auditory cortex, compared to the occipital, parietal, and frontal cortices. They further described coupled gene expression in a different gene group at a late mid-fetal stage in the superior and inferior temporal cortex and in the posterior inferior parietal cortex. Norm variations in these couplings might have caused atypical asymmetric folding of STS in the female fetus in our sample, which then triggered a cascade of subtle structural changes in the neo-cortex that consequently might have resulted in atypical language lateralization at the postfetal age of 7 years.

A further exploration of the localization of processing differences in the language paradigm between the atypically lateralized twin and the reference group revealed stronger activation in the left parahippocampal, fusiform, and lingual gyri. Increased activation of the inferior temporo-occipital junction areas was previously associated with processing of semantic category-specificity (Chan et al. 2011). The increased activity in the aforementioned cluster in the atypically lateralized twin might suggest qualitative variation in processing of the task we applied, which included semantic decisions. The stronger activation of the ventral temporooccipital junction could also suggest the stronger need for categorization of semantic contexts in the atypically developing brain.

Complementary differences between the atypically lateralized twin and her brother were obvious, not only in terms of language lateralization, but also in terms of functional 
connectivity. While seed voxel correlation of the left IFG seemed to result in a left-lateralized connectivity pattern in both twins, comparable to McAvoy et al. (2016), the right IFG seed revealed stronger local, contralateral IFG and ipsilateral remote connectivity in the atypical twin, compared to her brother. These findings might suggest that both twins had similar left-lateralized IFG connectivity, but the atypically lateralized twin showed additional stronger contralateral connectivity to a different part of the IFG. The posterior left STS seed voxel correlation also revealed stronger bilateral connectivity between the temporo-parietal junction (TPJ) regions in the atypically lateralized twin. Furthermore, the seed voxel correlation of the right STS comprised a much wider bilateral network of language areas in the female compared to the male twin. It could, therefore, be possible that both twins demonstrated equal leftward language processing, with the atypically lateralized twin having stronger crosshemispheric connections than the typical sample, resulting in a rightward lateralization shift during the language paradigm. This might have been the result of a qualitatively different processing mechanism of the contralateral hemisphere as a reaction to subtle variations in prenatal brain development. The role of contralateral involvement in association with recovery from brain damage was recently highlighted in a review by Bartolomeo and de Schotten (2016). We suppose that compensatory contralateral hemisphere involvement is also involved in more subtle structural norm variations.

One further point of discussion is the association between handedness and language lateralization. Although pleiotropy has been suggested for these two functions [compare Ocklenburg et al. (2014)], evidence suggests that the genetic basis for these phenomena is only partly overlapping (Somers et al. 2015) or even completely dissociable (Schmitz et al. 2017). Our study case that showed strong right-handedness herself as well as no familial left-handedness in combination with both developmental structural and functional rightward inverted laterality is a clear indicator for the complete dissociability between handedness and language lateralization.

Finally, it is arguable if postnatal laterality differences are partly caused by gender differences (Yu et al. 2014; Schmithorst and Holland 2007). However, we accounted for this issue by comparing the female atypically lateralized twin to a mixed-gendered control group. Since this comparison revealed highly significant language laterality differences in temporal and frontal lobes and former studies would suggest more a gradually weaker language lateralization pattern than a shift towards the right hemisphere in females (Nenert et al. 2017; Yu et al. 2014), we suppose this difference in laterality to be caused by more far-reaching biological mechanisms than exclusively gender differences. In addition to that it is quite mentionable that male-female twins represent a unique configuration in human development as investigated extensively by Boklage [e.g., Boklage (2009)]. It turned out that the female twin shows some specialties in neurocognitive and physical development caused by increased intrauterine testosterone exposure. However, it is questionable how these processes associated with 'masculinization' of a female would cause a completely inverted configuration of the fetal temporal lobes paired with specific atypical language organization at the age of seven. Further investigations on this topic are needed to uncover if these processes are related to each other or if the observation in our twin couple was by chance or due to other mechanisms associated with prenatal and postnatal development in twinship.

From these results, we conclude that interference in the processes included in the formation of the temporal lobes might be reflected in cognitive processing, as demonstrated by language lateralization, language localization, and resting-state connectivity over the course of childhood. However, we see no link between such processing differences and a broad range of cognitive skills to date.

The analysis for the forward speech condition revealed increased activations to the frontal midline, left hippocampus and left superior temporal sulcus, thus regions associated with language processing, memory retrieval and executive functioning. Specific to reverse speech was the increased activation of the precuneus and right supramarginal gyrus, which are regions associated with mind wandering, indicative of the default mode (Christoff et al. 2009, 2016; Andrews-Hanna 2012), and somatosensation, respectively.

Interestingly, Im et al. (2009) could show in an adult sample that the depth of the right STS is more variable than the left. Based on the fact that the fetal STS is already inherently deeper (Kasprian et al. 2011; Habas et al. 2012), there is also more room for variability; however, the fetal asymmetric folding pattern of the STS is a very common feature, which almost all fetuses show. It might be interesting to investigate how this structural pattern develops and its clearness fades over time.

Acknowledgements Open access funding provided by Austrian Science Fund (FWF). This work was supported by the Austrian Science Fund (FWF), Grant KLI 544-B27 and I 2714-B31. We thank Gerlinde M. Gruber for the maintenance of the fetal MR database and Peter Brugger for the acquisition of fetal MR data. We thank Mary McAllister for proofreading.

Funding This study was funded by the Austrian Science Fund (FWF; Grant KLI 544-B27 \& I 2714-B31).

\section{Compliance with ethical standards}

Ethical approval All procedures performed in studies involving human participants were in accordance with the ethical standards of the insti- 
tutional and/or national research committee and with the 1964 Helsinki declaration and its later amendments or comparable ethical standards.

Conflict of interest The authors declare that they have no conflict of interest.

Open Access This article is distributed under the terms of the Creative Commons Attribution 4.0 International License (http://creativeco mmons.org/licenses/by/4.0/), which permits unrestricted use, distribution, and reproduction in any medium, provided you give appropriate credit to the original author(s) and the source, provide a link to the Creative Commons license, and indicate if changes were made.

\section{References}

Andrews-Hanna JR (2012) The brain's default network and its adaptive role in internal mentation. Neuroscientist 18(3):251-270

Balsamo LM, Xu B, Gaillard WD (2006) Language lateralization and the role of the fusiform gyrus in semantic processing in young children. Neuroimage 31(3):1306-1314. https://doi. org/10.1016/j.neuroimage.2006.01.027

Bartolomeo P, de Schotten MT (2016) Let thy left brain know what thy right brain doeth: inter-hemispheric compensation of functional deficits after brain damage. Neuropsychologia 93:407-412

Beauchamp MS, Nath AR, Pasalar S (2010) fMRI-guided transcranial magnetic stimulation reveals that the superior temporal sulcus is a cortical locus of the McGurk effect. J Neurosci 30(7):2414-2417

Binder JR, Frost JA, Hammeke TA, Cox RW, Rao SM, Prieto T (1997) Human brain language areas identified by functional magnetic resonance imaging. J Neurosci 17(1):353-362

Boklage CE (2009) Traces of embryogenesis are the same in monozygotic and dizygotic twins: not compatible with double ovulation. Hum Reprod 24(6):1255-1266

Chan AM, Baker JM, Eskandar E, Schomer D, Ulbert I, Marinkovic K, Cash SS, Halgren E (2011) First-pass selectivity for semantic categories in human anteroventral temporal lobe. J Neurosci 31(49):18119-18129

Chi JG, Dooling EC, Gilles FH (1977) Gyral development of the human brain. Ann Neurol 1(1):86-93

Christoff K, Gordon AM, Smallwood J, Smith R, Schooler JW (2009) Experience sampling during fMRI reveals default network and executive system contributions to mind wandering. Proc Natl Acad Sci 106(21):8719-8724

Christoff K, Irving ZC, Fox KC, Spreng RN, Andrews-Hanna JR (2016) Mind-wandering as spontaneous thought: a dynamic framework. Nat Rev Neurosci 17(11):718-731

Crawford JR, Howell DC (1998) Comparing an individual's test score against norms derived from small samples. Clin Neuropsychol 12(4):482-486

Deen B, Koldewyn K, Kanwisher N, Saxe R (2015) Functional organization of social perception and cognition in the superior temporal sulcus. Cereb Cortex 25(11):4596-4609

Dehaene S, Dehaene-Lambertz G (2016) Is the brain prewired for letters? Nat Neurosci 19(9):1192-1193

Dobyns WB (1987) Developmental aspects of lissencephaly and the lissencephaly syndromes. Birth Defects Orig Artic Ser 23(1):225

Dorovini-Zis K, Dolman C (1977) Gestational development of brain. Arch Pathol Lab Med 101(4):192-195

Dorsaint-Pierre R, Penhune VB, Watkins KE, Neelin P, Lerch JP, Bouffard M, Zatorre RJ (2006) Asymmetries of the planum temporale and Heschl's gyrus: relationship to language lateralization. Brain 129(5):1164-1176
Dubois J, Benders M, Borradori-Tolsa C, Cachia A, Lazeyras F, HaVinh Leuchter R, Sizonenko S, Warfield S, Mangin J, Hüppi PS (2008) Primary cortical folding in the human newborn: an early marker of later functional development. Brain 131(8):2028-2041

Fedorenko E, Thompson-Schill SL (2014) Reworking the language network. Trends Cogn Sci 18(3):120-126

Fedorenko E, Nieto-Castanon A, Kanwisher N (2012) Lexical and syntactic representations in the brain: an fMRI investigation with multi-voxel pattern analyses. Neuropsychologia 50(4):499-513

Gaillard WD, Berl MM, Moore EN, Ritzl EK, Rosenberger LR, Weinstein SL, Conry JA, Pearl PL, Ritter FF, Sato S, Vezina LG, Vaidya CJ, Wiggs E, Fratalli C, Risse G, Ratner NB, Gioia G, Theodore WH (2007) Atypical language in lesional and nonlesional complex partial epilepsy. Neurology 69(18):17611771. https://doi.org/10.1212/01.wnl.0000289650.48830.1a

Geschwind N, Galaburda AM (1985) Cerebral lateralization: Biological mechanisms, associations, and pathology: I. A hypothesis and a program for research. Arch Neurol 42(5):428-459

Geschwind N, Levitsky W (1968) Human brain: left-right asymmetries in temporal speech region. Science 161(3837):186-187

Greve DN, Van der Haegen L, Cai Q, Stufflebeam S, Sabuncu MR, Fischl B, Brysbaert M (2013) A surface-based analysis of language lateralization and cortical asymmetry. J Cogn Neurosci 25(9):1477-1492. https://doi.org/10.1162/jocn_a_00405

Habas PA, Scott JA, Roosta A, Rajagopalan V, Kim K, Rousseau F, Barkovich AJ, Glenn OA, Studholme C (2012) Early folding patterns and asymmetries of the normal human brain detected from in utero MRI. Cereb Cortex 22(1):13-25

Hackman DA, Farah MJ (2009) Socioeconomic status and the developing brain. Trends Cogn Sci 13(2):65-73

Im K, Jo HJ, Mangin J-F, Evans AC, Kim SI, Lee J-M (2009) Spatial distribution of deep sulcal landmarks and hemispherical asymmetry on the cortical surface. Cereb Cortex 20(3):602-611

Josse G, Mazoyer B, Crivello F, Tzourio-Mazoyer N (2003) Left planum temporale: an anatomical marker of left hemispheric specialization for language comprehension. Brain Res Cogn Brain Res 18(1):1-14

Josse G, Kherif F, Flandin G, Seghier ML, Price CJ (2009) Predicting language lateralization from gray matter. J Neurosci 29(43):13516-13523. https://doi.org/10.1523/JNEUR OSCI.1680-09.2009

Kasprian G, Langs G, Brugger PC, Bittner M, Weber M, Arantes M, Prayer D (2011) The prenatal origin of hemispheric asymmetry: an in utero neuroimaging study. Cereb Cortex 21(5):1076-1083. https://doi.org/10.1093/cercor/bhq179

Keller SS, Roberts N, García-Fiñana M, Mohammadi S, Ringelstein EB, Knecht S, Deppe M (2011) Can the language-dominant hemisphere be predicted by brain anatomy? J Cogn Neurosci 23(8):2013-2029

Kersbergen KJ, Leroy F, Išgum I, Groenendaal F, de Vries LS, Claessens NH, van Haastert IC, Moeskops P, Fischer C, Mangin J-F (2016) Relation between clinical risk factors, early cortical changes, and neurodevelopmental outcome in preterm infants. Neuroimage 142:301-310

Knecht S, Deppe M, Drager B, Bobe L, Lohmann H, Ringelstein E, Henningsen $H$ (2000) Language lateralization in healthy righthanders. Brain 123(Pt 1):74-81

Leroy F, Cai Q, Bogart SL, Dubois J, Coulon O, Monzalvo K, Fischer C, Glasel H, Van der Haegen L, Benezit A, Lin CP, Kennedy DN, Ihara AS, Hertz-Pannier L, Moutard ML, Poupon C, Brysbaert M, Roberts N, Hopkins WD, Mangin JF, Dehaene-Lambertz G (2015) New human-specific brain landmark: the depth asymmetry of superior temporal sulcus. Proc Natl Acad Sci USA 112(4):12081213. https://doi.org/10.1073/pnas.1412389112 
Lidzba K, Schwilling E, Grodd W, Krägeloh-Mann I, Wilke M (2011) Language comprehension vs. language production: age effects on fMRI activation. Brain Lang 119(1):6-15

Lohmann G, Hoehl S, Brauer J, Danielmeier C, BornkesselSchlesewsky I, Bahlmann J, Turner R, Friederici A (2009) Setting the frame: the human brain activates a basic low-frequency network for language processing. Cereb Cortex 20(6):1286-1292

Li X, Crow TJ, Hopkins WD, Gong Q, Roberts N (2018) Human torque is not present in chimpanzee brain. NeuroImage 165:285-293

Mahmoudzadeh M, Dehaene-Lambertz G, Fournier M, Kongolo G, Goudjil S, Dubois J, Grebe R, Wallois F (2013) Syllabic discrimination in premature human infants prior to complete formation of cortical layers. Proc Natl Acad Sci 110(12):4846-4851

McAvoy M, Mitra A, Coalson RS, d'Avossa G, Keidel JL, Petersen SE, Raichle ME (2016) Unmasking language lateralization in human brain intrinsic activity. Cereb Cortex 26(4):1733-1746

Meynert T (1868) Der Bau der Gross-Hirnrinde: und seine örtlichen Verschiedenheiten, nebst einem pathologisch-anatomischen Corollarium. Heuser

Miller JA, Ding SL, Sunkin SM, Smith KA, Ng L, Szafer A, Ebbert A, Riley ZL, Royall JJ, Aiona K, Arnold JM, Bennet C, Bertagnolli D, Brouner K, Butler S, Caldejon S, Carey A, Cuhaciyan C, Dalley RA, Dee N, Dolbeare TA, Facer BA, Feng D, Fliss TP, Gee G, Goldy J, Gourley L, Gregor BW, Gu G, Howard RE, Jochim JM, Kuan CL, Lau C, Lee CK, Lee F, Lemon TA, Lesnar P, McMurray B, Mastan N, Mosqueda N, Naluai-Cecchini T, Ngo NK, Nyhus J, Oldre A, Olson E, Parente J, Parker PD, Parry SE, Stevens A, Pletikos M, Reding M, Roll K, Sandman D, Sarreal M, Shapouri S, Shapovalova NV, Shen EH, Sjoquist N, Slaughterbeck CR, Smith M, Sodt AJ, Williams D, Zollei L, Fischl B, Gerstein MB, Geschwind DH, Glass IA, Hawrylycz MJ, Hevner RF, Huang H, Jones AR, Knowles JA, Levitt P, Phillips JW, Sestan N, Wohnoutka P, Dang C, Bernard A, Hohmann JG, Lein ES (2014) Transcriptional landscape of the prenatal human brain. Nature 508(7495):199-206. https://doi.org/10.1038/nature13185

Nenert R, Allendorfer JB, Martin AM, Banks C, Vannest J, Holland SK, Szaflarski JP (2017) Age-related language lateralization assessed by fMRI: the effects of sex and handedness. Brain Res 1674:20-35

Ocklenburg S, Beste C, Arning L, Peterburs J, Gunturkun O (2014) The ontogenesis of language lateralization and its relation to handedness. Neurosci Biobehav Rev 43:191-198. https://doi. org/10.1016/j.neubiorev.2014.04.008

Oldfield RC (1971) The assessment and analysis of handedness: the Edinburgh inventory. Neuropsychologia 9(1):97-113

Plante E, Swisher L, Vance R (1989) Anatomical correlates of normal and impaired language in a set of dizygotic twins. Brain Lang 37(4):643-655

Pletikos M, Sousa AM, Sedmak G, Meyer KA, Zhu Y, Cheng F, Li M, Kawasawa YI, Šestan N (2014) Temporal specification and bilaterality of human neocortical topographic gene expression. Neuron 81(2):321-332

Poeppel D (2014) The neuroanatomic and neurophysiological infrastructure for speech and language. Curr Opin Neurobiol 28:142-149

Power JD, Barnes KA, Snyder AZ, Schlaggar BL, Petersen SE (2012) Spurious but systematic correlations in functional connectivity MRI networks arise from subject motion. Neuroimage 59(3):2142-2154
Rajagopalan V, Scott J, Habas PA, Kim K, Corbett-Detig J, Rousseau F, Barkovich AJ, Glenn OA, Studholme C (2011) Local tissue growth patterns underlying normal fetal human brain gyrification quantified in utero. J Neurosci 31(8):2878-2887

Redcay E (2008) The superior temporal sulcus performs a common function for social and speech perception: implications for the emergence of autism. Neurosci Biobehav Rev 32(1):123-142

Rickham P (1964) Human experimentation. Code of ethics of the world medical association. Declar Hels Br Med J 2(5402):177-177

Schmithorst VJ, Holland SK (2007) Sex differences in the development of neuroanatomical functional connectivity underlying intelligence found using Bayesian connectivity analysis. Neuroimage 35(1):406-419

Schmitz J, Lor S, Klose R, Güntürkün O, Ocklenburg S (2017) The functional genetics of handedness and language lateralization: insights from gene ontology, pathway and disease association analyses. Front Psychol. https://doi.org/10.3389/fpsyg.2017.01144

Schuler A-L, Kasprian G, Schwartz E, Seidl R, Diogo MC, Mitter C, Langs G, Prayer D, Bartha-Doering L (2017) Mens inversus in corpore inverso? Language lateralization in a boy with situs inversus totalis. Brain Lang 174:9-15

Sepeta LN, Berl MM, Wilke M, You X, Mehta M, Xu B, Inati S, Dustin I, Khan O, Austermuehle A (2016) Age-dependent mesial temporal lobe lateralization in language fMRI. Epilepsia 57(1):122-130

Somers M, Ophoff RA, Aukes MF, Cantor RM, Boks MP, Dauwan M, de Visser KL, Kahn RS, Sommer IE (2015) Linkage analysis in a Dutch population isolate shows no major gene for left-handedness or atypical language lateralization. J Neurosci 35(23):8730-8736

Sun T, Patoine C, Abu-Khalil A, Visvader J, Sum E, Cherry TJ, Orkin SH, Geschwind DH, Walsh CA (2005) Early asymmetry of gene transcription in embryonic human left and right cerebral cortex. Science 308(5729):1794-1798

Tzourio N, Nkanga-Ngila B, Mazoyer B (1998) Left planum temporale surface correlates with functional dominance during story listening. Neuroreport 9(5):829-833

Vigneau M, Beaucousin V, Herve P-Y, Duffau H, Crivello F, Houde O, Mazoyer B, Tzourio-Mazoyer N (2006) Meta-analyzing left hemisphere language areas: phonology, semantics, and sentence processing. Neuroimage 30(4):1414-1432

Wada JA, Clarke R, Hamm A (1975) Cerebral hemispheric asymmetry in humans: cortical speech zones in 100 adult and 100 infant brains. Arch Neurol 32(4):239-246

Wernicke C (1874) Der aphasische Symptomencomplex: eine psychologische Studie auf anatomischer Basis. Cohn

Wilke M, Lidzba K (2007) LI-tool: a new toolbox to assess lateralization in functional MR-data. J Neurosci Methods 163(1):128-136. https://doi.org/10.1016/j.jneumeth.2007.01.026

Wilke M, Schmithorst VJ (2006) A combined bootstrap/histogram analysis approach for computing a lateralization index from neuroimaging data. Neuroimage 33(2):522-530

You X, Adjouadi M, Guillen MR, Ayala M, Barreto A, Rishe N, Sullivan J, Dlugos D, Vanmeter J, Morris D, Donner E, Bjornson B, Smith ML, Bernal B, Berl M, Gaillard WD (2011) Sub-patterns of language network reorganization in pediatric localization related epilepsy: a multisite study. Hum Brain Mapp 32(5):784-799. https ://doi.org/10.1002/hbm.21066

Yu VY, MacDonald MJ, Oh A, Hua GN, De Nil LF, Pang EW (2014) Age-related sex differences in language lateralization: a magnetoencephalography study in children. Dev Psychol 50(9):2276 